\title{
Use of cotton as a sorbent for on-line precolumn enrichment of polycyclic aromatic hydrocarbons in waters prior to liquid chromatography determination
}

\author{
Jing-fu Liu ${ }^{\mathrm{a}}$, Yu-guang Chi ${ }^{\mathrm{a}, \mathrm{b}}$, Gui-bin Jiang ${ }^{\mathrm{a}, *}$, Chao Tai ${ }^{\mathrm{a}}$, Jing-tian $\mathrm{Hu}^{\mathrm{b}}$ \\ ${ }^{\mathrm{a}}$ Key Laboratory of Environmental Chemistry and Ecotoxicology, Research Center for Eco-Environmental Sciences, \\ Chinese Academy of Sciences, P.O. Box 2871, Beijing 100085, PR China \\ ${ }^{\mathrm{b}}$ Department of Chemistry, Shandong University, Jinan 250100, PR China
}

Received 5 July 2003; received in revised form 10 September 2003; accepted 11 September 2003

\begin{abstract}
Using cotton as a solid-phase extraction sorbent of the precolumn, an on-line coupled precolumn preconcentration-liquid chromatography system with fluorescence detection was developed for the determination of PAHs in aqueous samples. Four PAHs including fluorene, phenanthrene, fluoranthene and benzo $[k]$ fluoranthene were preconcentrated by a precolumn packed with 30 $\mathrm{mg}$ of absorbent cotton and then separated by $\mathrm{C}_{18}$ analytical column. When $100 \mathrm{ml}$ of sample was enriched, the proposed procedure provided detection limits in the range of $0.5-57 \mathrm{ng} \mathrm{1^{-1 }}$. Several water samples spiked with PAHs were analyzed with recoveries in the range of $92-119 \%$ at spiking level of $100 \mathrm{ng} \mathrm{1^{-1 }}$ for fluorene, phenanthrene and fluoranthene, and $10 \mathrm{ng} 1^{-1}$ for benzo $[k]$ fluoranthene, respectively.
\end{abstract}

(c) 2003 Elsevier B.V. All rights reserved.

Keywords: Cotton; Precolumn; Liquid chromatography

\section{Introduction}

Polycyclic aromatic hydrocarbons (PAHs) are a group of organic compounds, which have received considerable attention because of their carcinogenicity, mutagenicity, toxicity and environmental ubiquitousness. Although the analytical instrumentation such as gas chromatography with mass spectrometry detection (GC/ $\mathrm{MS}$ ) and high performance liquid chromatography with fluorescence detection (HPLC/FLD) for this purpose has improved dramatically, determination of trace levels of PAHs in aqueous samples is still a challenge as sample preconcentration and/or clean up is usually required [1-3].

Although some new techniques including solid-phase micro-extraction (SPME) [4,5] and liquid-phase microextraction [6] have been reported for the extraction of PAHs from water, liquid-liquid extraction (LLE) and solid-phase extraction (SPE) are the most commonly used techniques for enriching and cleaning of PAHs

\footnotetext{
*Corresponding author. Fax: + 86-10-62849179.

E-mail address: gbjiang@mail.rcees.ac.cn (G.-b. Jiang).
}

from aqueous samples [3]. Because LLE has many wellknown disadvantages such as emulsion and large volume consumption of toxic and flammable solvents, SPE are more frequently used in recent years [1-3].

Many commonly used sorbents including $\mathrm{C}_{18}, \mathrm{C}_{8}$, cyano and phenyl bonded silica sorbents have been used in the SPE of PAHs, but the most commonly used ones are the $\mathrm{C}_{18}$-bonded silica sorbents [2] and styrenedivinylbenzene copolymers like PLRP-s [7]. Various selective sorbents, predominantly the copper phthacyanine trisulfonate type, were also proposed for extracting PAHs [2,3]. In recent years, anti-fluorene [8-10] and anti-pyrene [11] immunosorbents have been proposed as alternatives to highly selective SPE of PAHs from aqueous, sediment or human urine samples. Solid cyclodextrin was also proposed for extraction of volatile PAHs from air [12].

It was reported that some natural fibers have higher sorption capacities for oil than commercially available synthetic fibers [13]. Choi et al. demonstrated that cotton, milkweed, and kenaf have 1.5-3 times better sorption properties than polypropylene fibers [14,15]. 
Table 1

Fluorescence detection conditions for HPLC determination of PAHs

\begin{tabular}{lcll}
\hline Analyte & Time $(\mathrm{min})$ & \multicolumn{2}{l}{ Fluorescence $(\mathrm{nm})$} \\
\cline { 3 - 4 } & & Excitation & Emission \\
\hline Fluorene & 0 & 250 & 440 \\
Phenanthrene & 13.00 & 215 & 320 \\
Fluoranthene & 16.00 & 250 & 360 \\
Benzo[k]fluoranthene & 20.00 & 234 & 445 \\
& & 250 & 425 \\
\hline
\end{tabular}

Because of their excellent oil sorption properties and high biodegradability, wool-based non-woven material [16] and hydrophobic cotton fibers [17] were adopted to remove oil from water. Recently, cotton column were used to selectively retain synthetic colorants [18]. These above reports indicated that cotton fibers were excellent sorbents for hydrophobic compounds. To our knowledge, however, no study on using cotton fibers as sorbents for preconcentration of hydrophobic compounds such as PAHs has been reported.

In this present study, an on-line coupled SPE-HPLC system was developed for determination of PAHs in water samples. PAHs were enriched by on-line SPE with cotton as a sorbent followed by HPLC determination with fluorescence detection.

\section{Experimental}

\subsection{Reagents and material}

Individual benzo[ $k]$ fluoranthene standard, and mixed standard of fluorene, phenanthrene and fluoranthene were obtained from the Institute for Reference Materials of SEPA (Beijing, China). HPLC-grade acetonitrile were purchased from Scharlace Chemie SA, Barcelona, Spain. All other chemicals were of analytical grade (Beijing Chemicals Corporation, Beijing, China) and ultrapure water (EASYpure LF) was used throughout. Cotton wool (Beijing Lingrui Sanitary Material Co.) was the one commonly used in hospital, and was pretreated by washing with acetonitrile before use.

\subsection{Instrument}

The HPLC equipment used included an Agilent 1100 Series BinPump, and a FLD detector. For all compounds of interest the excitation and the emission wavelength of the fluorescence detector was set as shown in Table 1. A personal computer equipped with an Agilent ChemStation program for LC systems was used to process the chromatographic data. An Agilent Zorbax RX- $\mathrm{C}_{8}$ column $(150 \times 4.6 \mathrm{~mm}$, particle size $5 \mu \mathrm{m})$ was used as analytical column. Samples were injected with a 7725 injector (Rheodyne, USA) whose sampling loop was replaced by a precolumn (HPLC sample clean-up and guard column packed with $30 \mathrm{mg}$ of absorbent cotton, $20 \times 2 \mathrm{~mm}$ I.D.) where analytes were extracted.

The chromatographic separation was performed with a mobile phase consisted of water and acetonitrile delivered at a flow rate of $1.0 \mathrm{ml} \mathrm{min}{ }^{-1}$. The gradient program was as follows: constant $54 \%$ mixture of acetonitrile during $0-20 \mathrm{~min}$, then increasing to $90 \%$ in $2 \mathrm{~min}$, and thereafter restored to $54 \%$ in $3 \mathrm{~min}$.

\subsection{Extraction procedure}

Samples, acetonitrile and water were delivered by a piston pump (MiniPump, Laboratory Date Control, Division of Milton Roy Company) at $3.0 \mathrm{ml} \mathrm{min}{ }^{-1}$. The precolumn was first washed with $5 \mathrm{ml}$ of acetonitrile and water successively. Then, $100 \mathrm{ml}$ of sample, containing 10\% 2-propanol, was introduced onto the precolumn. After washing the precolumn with $5 \mathrm{ml}$ of water, the analytes focused on the precolumn were injected into the chromatographic system by eluting in back-flush mode with the mobile phase.

\subsection{Sampling}

Tap water sample was collected from water tap in our laboratory, river water samples were collected from the Yongding river valley in the section of Beijing, and the wastewater sample was the effluent collected at Gaobeidian sewage treatment works in the east part of Beijing. Samples were collected in cleaned glass bottles and $10 \%$ of 2-propanol was added to stop the compounds from being adsorbed onto the wall of the bottles. The samples were then filtered through cellulose membrane with pore size of $0.45 \mu \mathrm{m}$ and maintained in glass containers, then stored at a temperature of $4{ }^{\circ} \mathrm{C}$.

\section{Results and discussion}

\subsection{Optimization of on-line precolumn preconcentration process}

The influence of some parameters for on-line preconcentration of PAHs were investigated by enriching a mixing standard containing $100 \mathrm{ng}^{-1}$ of fluorene, phenanthrene, fluoranthene, and $10 \mathrm{ng} 1^{-1}$ of benzo[ $k]$ fluoranthene as model compounds. Each experiment was repeated three times and the data shown in this study were the mean values.

\subsubsection{2-Propanol concentration}

In SPE of PAHs, organic solvents such as 2-propanol concentration or acetonitrile are usually added into sample solutions to prevent the adsorbing of PAHs onto the walls and surfaces of the system. The effect of 2propanol concentration on the analytical signal was 


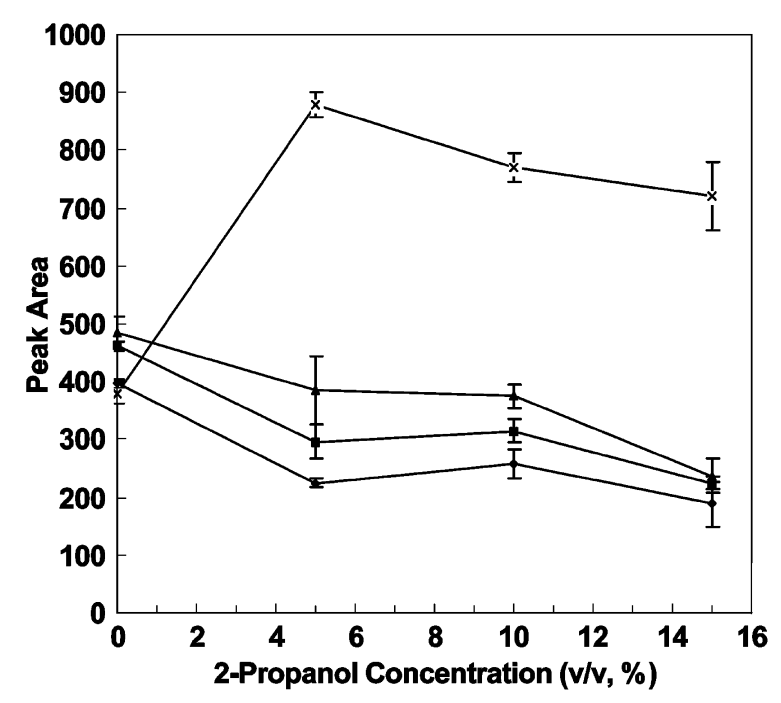

Fig. 1. Effect of 2-propanol concentration on the peak area of PAHs when $60 \mathrm{ml}$ of mixing standards were preconcentrated., 100

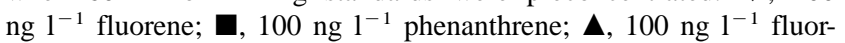
anthene; $\times, 10 \mathrm{ng}^{-1}$ benzo[ $k$ ]fluoranthene.

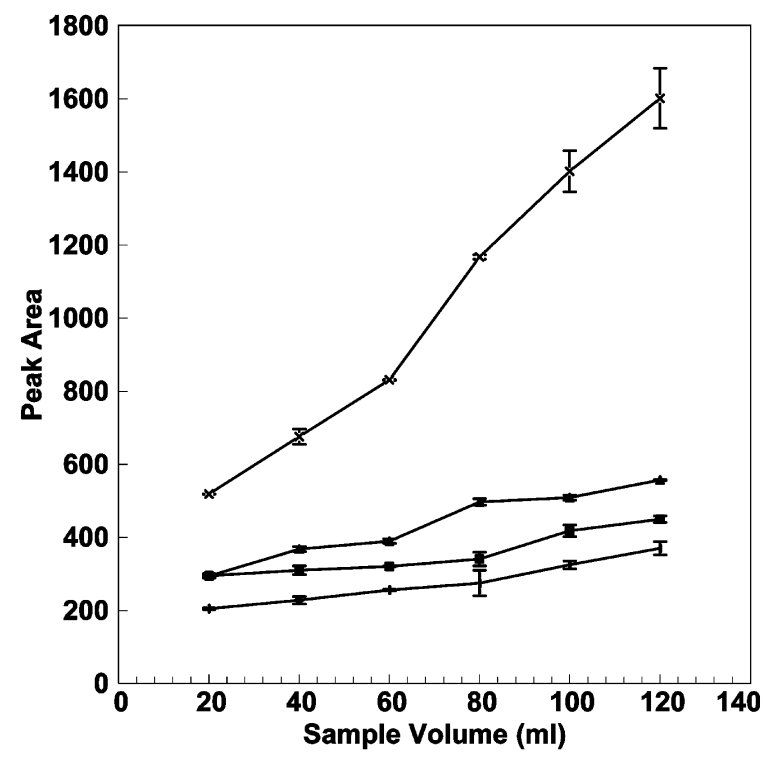

Fig. 2. Effect of sample volume on the peak area of PAHs. $\downarrow 100$ $\mathrm{ng}^{-1}$ fluorene; $\boldsymbol{\square}, 100 \mathrm{ng}^{-1}$ phenanthrene; $\boldsymbol{\Delta}, 100 \mathrm{ng}^{-1}$ fluoranthene; $\times, 10 \mathrm{ng}^{-1}$ benzo[k]fluoranthene.

Table 2

Some analytical performance date of the proposed method

\begin{tabular}{lllllr}
\hline Analyte & $\begin{array}{l}\text { Linear range } \\
\left.(\mathrm{ng} \mathrm{1})^{-1}\right)\end{array}$ & $\begin{array}{l}\text { Regression } \\
\text { Equation }\end{array}$ & $\begin{array}{l}\text { Correlation } \\
\text { coefficient }\left(R^{2}\right)\end{array}$ & $\begin{array}{l}\text { Reproducibility } \\
(\text { R.S.D.\%, } n=6)\end{array}$ & $\begin{array}{l}\text { Detection limit } \\
\left(\mathrm{ng} 1^{-1}\right)\end{array}$ \\
\hline Fluorene & $10-500$ & $y=4.468 x+14.25$ & 0.9995 & 13.5 & 57 \\
Phenanthrene & $10-500$ & $y=2.937 x+42.08$ & 0.9995 & 4.1 & 29 \\
Fluoranthene & $10-500$ & $y=2.221 x+21.43$ & 0.9994 & 3.0 & 5 \\
Benzo[k] & $0.1-5$ & $y=43.04 x+53.36$ & 0.9662 & 1.8 & 0.5 \\
$\quad$ fluoranthene & & & & &
\end{tabular}

investigated and results were shown in Fig. 1, which indicates the addition of 2-propanol decreasing the peak area of low molecular weight PAHs (fluorene, phenanthrene and fluoranthene) but significantly increased the peak area of high molecular weight PAHs (ben$\mathrm{zo}[k]$ fluoranthene). This is because 2-propanol can significantly increase the solubility of high molecular weight PAHs thus prevent its adsorbing on the walls and surfaces of the system. For the low molecular weight PAHs, however, 2-propanol can significantly decrease the recoveries by precolumn absorbents. Therefore, $10 \%$ of 2-propanol was adopted as a compromise in the following studies.

\subsubsection{Sample flow rate}

In on-line SPE system, the flow rate of the sample solution is one of the most important parameters, which affects both the recoveries of analytes and the time of sample preconcentration. The effect of sample flow rate was studied in the range of $0.7-3.0 \mathrm{ml} \mathrm{min}^{-1}$ and results showed that the peak areas kept unchanging, i.e. the flow rate of sample loading had no effect on the recoveries of the four analytes. However, higher flow rate of sample solutions resulted in unstable system because of the high back-pressure of cotton. Therefore, $3.0 \mathrm{ml} \mathrm{min}{ }^{-1}$ was adopted in the following studies.

\subsubsection{Sample volume}

Breakthrough volume is another one of the most important parameters that influence the concentration factor and reliability of analytical results of an on-line SPE system. It is very important to get satisfactory recoveries for all analytes in as large volume of sample solutions as possible. The effects of sample volumes on the peak area of the four analytes were studied in the range of 20-120 ml and results are shown in Fig. 2. As can be seen, the peak areas of all the studied PAHs increased with the increasing of sample volume. A sample volume of $100 \mathrm{ml}$ was recommended for analysis of real water samples.

\subsection{Analytical performance}

Some characters of the proposed method such as linear ranges, regression equations, correlation coefficients and detection limits were investigated by enrich- 
Table 3

Recoveries of PAHs in real water samples at spiking level of $100 \mathrm{ng} \mathrm{1^{-1 }}$ for fluorene, phenanthrene and fluoranthene, and $10 \mathrm{ng} 1^{-1}$ for benzo $[k]$ fluoranthene

\begin{tabular}{lcccc}
\hline Sample & \multicolumn{2}{l}{ Recovery $(\%$, Mean $\pm \mathrm{s}, n=3)$} & & \\
\cline { 2 - 5 } & Fluorene & Phenanthrene & Fluoranthene & Benzo $[k] f l u o r a n t h e n e$ \\
\hline Tap water & $92 \pm 3$ & $93 \pm 8$ & $93 \pm 1$ & $112 \pm 4$ \\
River water & $112 \pm 5$ & $119 \pm 3$ & $110 \pm 2$ & $106 \pm 6$ \\
Wastewater & $94 \pm 3$ & $98 \pm 2$ & $98 \pm 7$ & $118 \pm 2$ \\
\hline
\end{tabular}

ing $100 \mathrm{ml}$ of PAHs standard solutions and results were shown in Table 2.

Regression equation and correlation coefficient were obtained by determining five standards covering the linear range of each PAHs. The precision (R.S.D.) of the system was measured by six repeated determination of a standard solution containing $100 \mathrm{ng} \mathrm{1^{-1 }}$ each of fluorene, phenanthrene and fluoranthene, and $10 \mathrm{ng} \mathrm{l}^{-1}$ of benzo[ $[k]$ fluoranthene. The detection limits were calculated as $S / N=3$.

\subsection{Application to aqueous samples analysis}

To validate the proposed method, three aqueous samples including tap water, river water and wastewater (municipal sewage effluent) were analyzed and results showed that the contents of PAHs in these studied samples were all under the detection limits. The recoveries were determined by spiking the samples with 100 $\mathrm{ng} 1^{-1}$ each of fluorene, phenanthrene and fluoranthene, and $10 \mathrm{ng}^{-1}$ of benzo[k]fluoranthene, respectively. Results shown in Table 3 indicate that the recoveries were in the range of $92-119 \%$.

\section{Conclusion}

It was found that cotton could be used as sorbents for on-line precolumn preconcentration of PAHs prior to HPLC determination with fluorescence detection. Compared to the commonly used $\mathrm{C}_{18}$ sorbents, this new sorbent is very low-cost and very easy to be packed in a precolumn. For the four studied PAHs, the proposed procedure provided detection limits of $0.5-57 \mathrm{ng}^{-1}$ when $100 \mathrm{ml}$ of sample was enriched. The proposed procedure was applied to the determination of PAHs in various water samples with recoveries in the range of 93-119\% at spiking level of $100 \mathrm{ng}^{-1}$ for fluorene, phenanthrene and fluoranthene, and $10 \mathrm{ng} 1^{-1}$ for benzo[ $k]$ fluoranthene, respectively.

\section{Acknowledgments}

This work was supported by the National Natural Science Foundation of China $(20177026,20137010)$, Chinese Academy of Sciences (KZCX 2-410) and the State High Tech Development Plan (2002AA641010).

\section{References}

[1] E. Manoli, C. Samara, Trends Anal. Chem. 18 (1999) 417.

[2] R.M. Marce, F. Borrull, J. Chromatogr. A 855 (2000) 273.

[3] E.R. Brouwer, A.N.J. Hermans, H. Lingeman, U.A.Th. Brinkman, J. Chromatogr. A 669 (1994) 45.

[4] D.W. Potter, J. Pawliszyn, Environ. Sci. Technol. 28 (1994) 298.

[5] M.R. Negrao, M.F. Alpendurada, J. Chromatogr. A 823 (1998) 211.

[6] L. Zhao, H.K. Lee, Anal. Chem. 74 (2002) 2486-2492.

[7] R.A. Gimeno, A.F.M. Altelaar, R.M. Marce, F. Borrull, J. Chromatogr. A 958 (2002) 141.

[8] M. Bouzige, V. Pichon, M.-C. Hennion, J. Chromatogr. A 823 (1998) 197.

[9] N. Delaunay, V. Pichon, M.-C. Hennion, J. Chromatogr. B 745 (2000) 15.

[10] S. Perez, I. Ferrer, M.-C. Hennion, D. Barcelo, Anal. Chem. 70 (1998) 4996.

[11] M. Schedl, G. Wilharm, S. Achatz, A. Kettrup, R. Niessner, D. Knopp, Anal. Chem. 73 (2001) 5669.

[12] M.T. Butterfield, R.A. Agbaria, I.M. Warner, Anal. Chem. 68 (1996) 1187.

[13] R.F. Johnson, T.G. Manjrekar, J.E. Halligan, Environ. Sci. Technol. 7 (1973) 439.

[14] H.M. Choi, H.J. Kwon, J.P. Moreau, Text. Res. J. 63 (1993) 211.

[15] H.M. Choi, R.M. Cloud, Environ. Sci. Technol. 26 (1992) 772.

[16] M.M. Radetic, D.M. Jocic, P.M. Jovancic, Z. LJ. Petrovic, H.F. Thomas, Environ. Sci. Technol. 37 (2003) 1008.

[17] G. Deschamps, H. Caruel, M.-E. Borredon, C. Bonnin, C. Vignoles, Environ. Sci. Technol. 37 (2003) 1013.

[18] M. Gonzalez, M. Gallego, M. Valcarcel, Anal. Chem. 75 (2003) 685. 